\title{
The Planetary Nebulae in the Sagittarius Dwarf Galaxy
}

\author{
A. A. Zijlstra, G. Dudziak and J. R. Walsh
}

European Southern Observatory

There are 9 dwarf spheroidal galaxies in the neighbourhood of the Galaxy. The last to be discovered is in Sagittarius (Ibata et al 1994), situated at a distance of $25 \mathrm{kpc}$ and is interacting with the Galaxy. Its mass is $\geq 10^{7} \mathrm{M}_{\odot}$ making it perhaps the largest local dwarf spheroidal. The only other dwarf spheroidal known to contain a planetary nebula was Fornax (Danziger et al 1978).

Radial velocities of $\sim 50$ of Galactic Centre PN were obtained with the ESO 1.4m CAT and Coudé Echelle Spectrograph and several were found to be in the vicinity of the Sagittarius dwarf. Comparison of their radial velocity with that of Sagittarius $\left(+140 \mathrm{kms}^{-1}\right.$ dispersion $10 \mathrm{kms}^{-1}$ ) showed that He 2-436 (004.8-22.7) and Wray 16-423 (006.8-19.8) were very probable members of this galaxy (Zijlstra \& Walsh 1996).

Low dispersion spectra of both PN were obtained with EMMI on the NTT and the B\&C spectrograph on the ESO $1.5 \mathrm{~m}$. He 2-436 has a high density and the central star is of WC type. Wray $16-423$ is of lower density but higher ionization and has a very weak WC features. Abundances were derived from the forbidden lines and are listed in the Table. Whilst there are differences between the abundances of the two Sagittarius PN the values bracket those for the Fornax PN (Danziger et al 1978). For comparison, the abundances of the very metal poor halo PN DDDM-1 (Clegg et al (1987), the average Galactic PN (Aller \& Czyzak 1983) and solar abundances are also listed. The PN in dwarf spheroidals hold an important place in the study of the star formation history of these systems.

\begin{tabular}{lcccccc} 
Element & He 2-436 & Wray 16-423 & $\begin{array}{c}\text { Fornax } \\
\text { PN }\end{array}$ & DDDM-1 & $\begin{array}{c}\text { Galactic } \\
\text { PN }\end{array}$ & Solar \\
\hline $\mathrm{N}$ & 7.2 & 7.7 & 7.4 & 7.4 & 8.11 & 7.96 \\
$\mathrm{O}$ & 8.3 & 8.4 & 8.5 & 8.1 & 8.62 & 8.87 \\
$\mathrm{Ne}$ & 7.5 & 7.7 & 7.6 & 7.3 & 8.02 & 8.05 \\
$\mathrm{~S}$ & 6.3 & 6.5 & & 6.5 & 6.99 & 7.23 \\
$\mathrm{Ar}$ & 5.7 & 6.0 & 5.9 & 5.8 & 6.40 & 6.57
\end{tabular}

\section{REFERENCES}

Aller, L. H., Czyzak, S. J., 1983, ApJS, 51, 211

Clegg, R. E. S., Peimbert, M., Torres-Peimbert, S., 1987, MNRAS, 224, 761

Danziger, I. J., Dopita, M. A., Hawarden, T. G., Webster, B. L., 1978, ApJ, 220, 458

Ibata, R. A., Gilmore, G., Irwin, M. J., 1994, Nature, 370, 194

Zijlstra, A. A., Walsh, J. R., 1996, A\&A, 312, L21 

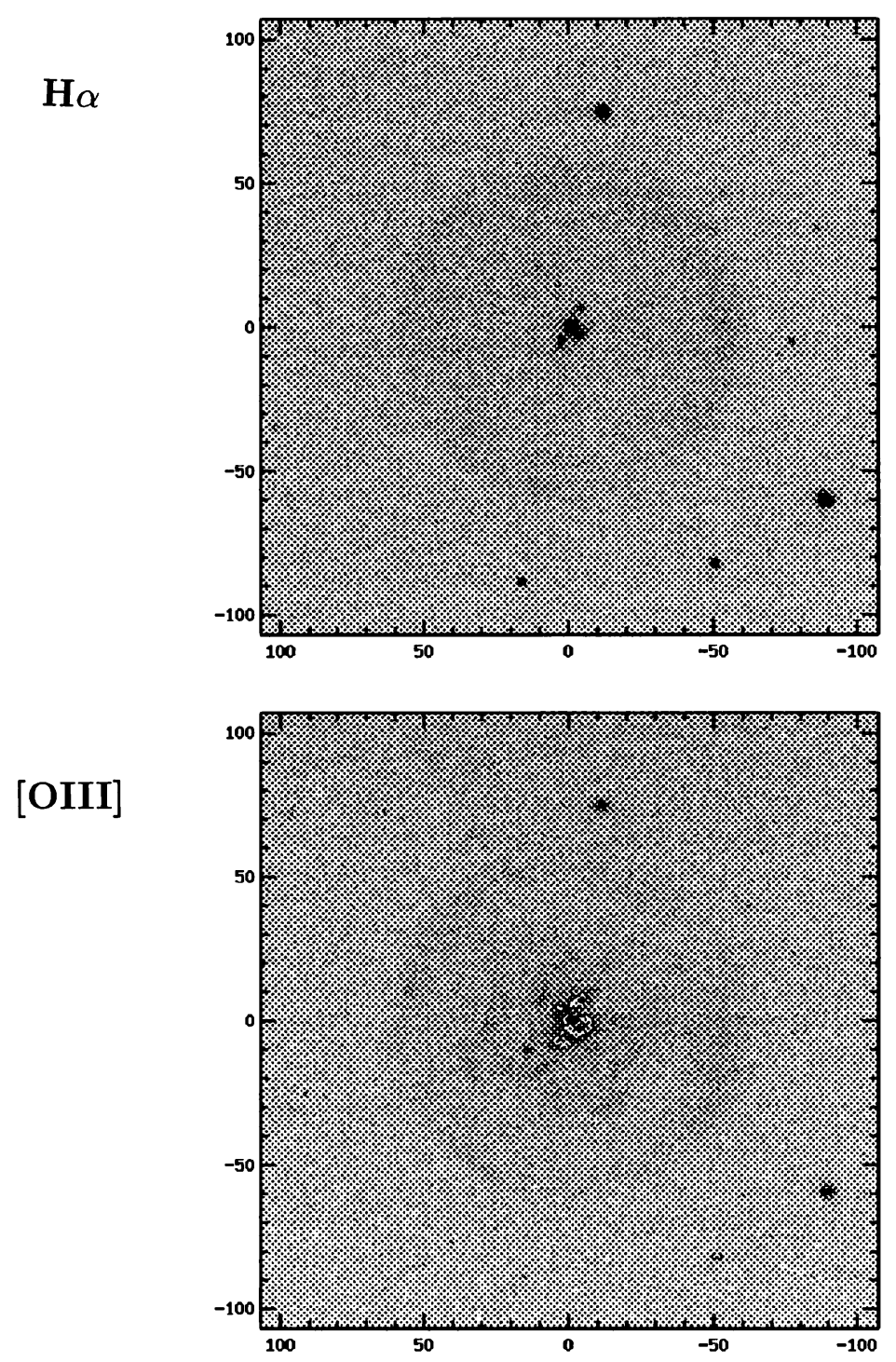

A $30208.5+33.2$

From: "The IAC Morphological Catalog of Northern Galactic Planetary Nebulae", A. Manchado, M.A. Guerrero, L. Stanghellini, M. Serre-Ricart. courtesy: A. Manchado 